www.conferenceie.ase.ro

\title{
AN ANALYSIS OF SCIENTIFIC PUBLICATIONS ON "DECISION SUPPORT SYSTEMS" AND "BUSINESS INTELLIGENCE" REGARDING RELATED CONCEPTS USING NATURAL LANGUAGE PROCESSING TOOLS
}

\author{
Daniel HOMOCIANU \\ Alexandru Ioan Cuza University of Iasi (UAIC), Romania \\ dan.homocianu@gmail.com \\ Alexandru SIRETEANU \\ Alexandru Ioan Cuza University of Iasi (UAIC), Romania \\ salexis@gmail.com \\ Octavian DOSPINESCU \\ Alexandru Ioan Cuza University of Iasi (UAIC), Romania \\ doctav@uaic.ro \\ Dinu AIRINEI \\ Alexandru Ioan Cuza University of Iasi (UAIC), Romania \\ adinu@uaic.ro
}

\begin{abstract}
In this paper we have analyzed scientific data regarding Decision Support Systems and Business Intelligence available on an online platform, namely Web of Science (apps.webofknowledge.com), in the Core Collection database. Starting from individual blocks consisting in concatenations of abstracts, titles and keywords, we extracted related concepts and corresponding importance scores using IBM Watson and a custom API. The results highlight the common points of the two topics being analyzed. They also reveal a much longer history of Business Intelligence, whose beginnings are usually placed almost 15 years later (e.g. Howard Dresner, 1989). Next we have plans to find the publication frequency considering both sets of related concepts and using the same Web of Science Core Collection database, and further perform analyzes of the resulting multiple time series using Data Mining tools.
\end{abstract}

Keywords: Decision Support Systems (DSS), Business Intelligence (BI), Web of Science (WOS) Core Collection database, Natural Language Processing (NLP), SQL, Data Mining JEL classification: D80, L86, C32

DOI: $10.12948 / \mathrm{ie} 2019.03 .03$

\section{Introduction}

There are many article studying the origins and use of Information Systems for managers such as DSS and BI. The literature from this specific field is also full of definitions and classifications but, with few exceptions, most of them are not able to provide consistent views not even for related concepts when considering a larger basis. There are also many contradictions such as the one regarding the theoretical and practical origins of the BI buzz-word. In the literature, there are many articles trying to synthetize the evolution of BI [1], [2], [3] and DSS [4], [5], [6]. In this article, our goal is to clarify the evolution of high quality publications in the field of DSS and BI and identify strong common points. 
www.conferenceie.ase.ro

\section{Data and main methods}

We have used data from the WOS Core Collection database. We extracted it in mid-March, current year (2019), under the form of almost 1100 full records for BI and approximately 7800 for DSS after filtering on relevance (only Science Citation Index Expanded - SCIE and Social Sciences Citation Index - SSCI in terms of WOS Index and articles, book chapters, proceedings papers published as articles in journals, and reviews in terms of Publication Type). This data as individual blocks consisting in title, abstracts and keywords were sent for analysis to IBM Watson using a custom Node JS API aiming for the extraction of maximum eight related concepts and corresponding scores per block in a way similar to the behavior of the IBM's Natural Language Processing demo. We first implemented a customized crawler because web crawling technologies help collecting and extracting large amounts of data from a lot of online sources. They are critical in web traffic analysis, cyber security, and in any area where data needs to be collected from multiple sources and analyzed in real time. The solution provided by us contains an automatic crawling mechanism, a human-user behavior emulation, and an automatic filling/check of 'refine results' forms from WOS with convenient controls for end users. User-generated content is the core element of concepts analysis in IBM Watson and this type of information could be easily acquired by deploying multiple web crawlers programmed to crawl data from different sources.

\section{Primary results and discussions}

The retrieval of primary scientific data after searching on both expressions indicated in the title of this paper can be done directly using the WOS platform, considering exports of maximum 500 records at a time.

Some abstracts were first cleaned of some expressions such as "Copyright" and the associated year that would have wrongly generated misleading concepts. To do that we have used some spreadsheet functions such as logical ones (IFERROR) and text-oriented ones (SEARCH, LEFT, MID, and LEN).

Next we involved the custom API developed in Node JS to retrieve concepts and corresponding importance scores from IBM Watson (Figure 1).

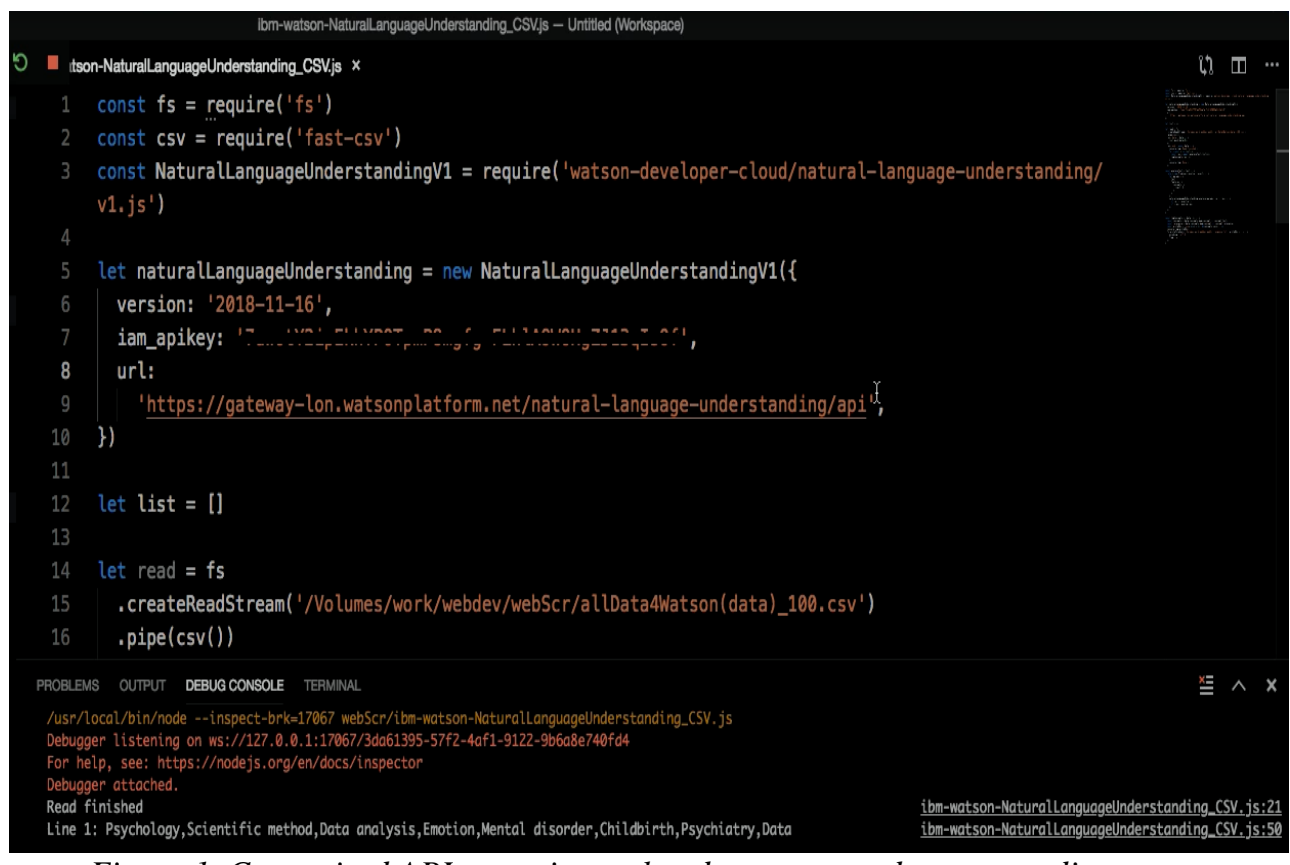

Figure 1. Customized API to retrieve related concepts and corresponding scores from IBM Watson 
After following these previous design and implementation steps, we applied the TRIM spreadsheet function to clean the items starting or ending in spaces. Then we divided the results into eight separate columns (Figure 2) for concepts and corresponding weights. Right after that split we sorted by the level 1 concept in order to eliminate the blank lines if existing.

Next we imported the resulting two spreadsheets with data in Access as two different tables (for both DSS and BI) and applied three types of SQL queries (listing 1): eight intermediate ones for computing the scores for distinct level i concepts, an aggregation one as a gross union with concept duplicates because of different levels involved and a final query to get the final ranking (Figure 3) considering all maximum eight levels (Figure 2) and distinct overall concepts.

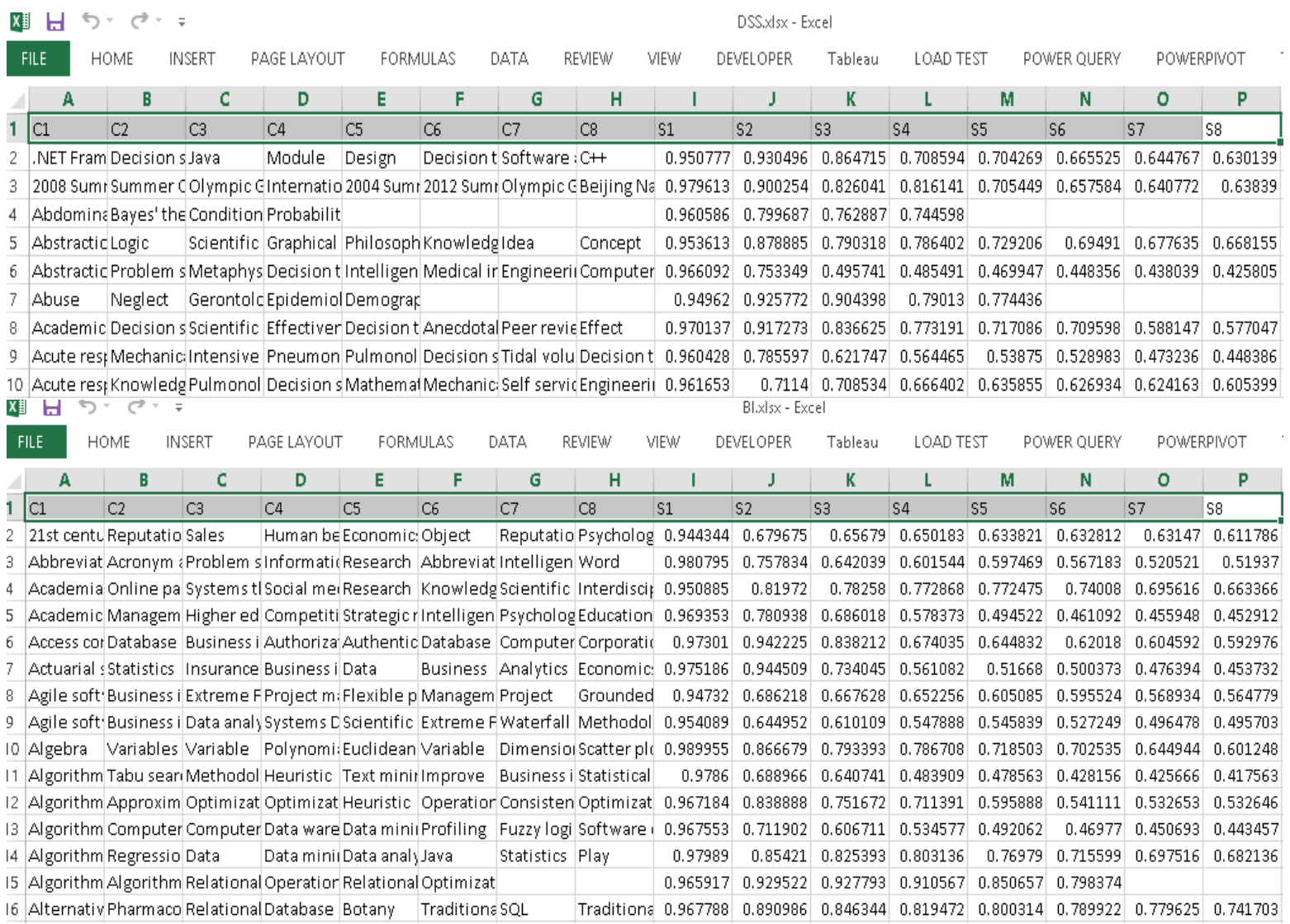

Figure 2. Further processed tabular data as distinct columns with concepts and scores / weights

Listing 1. SQL queries

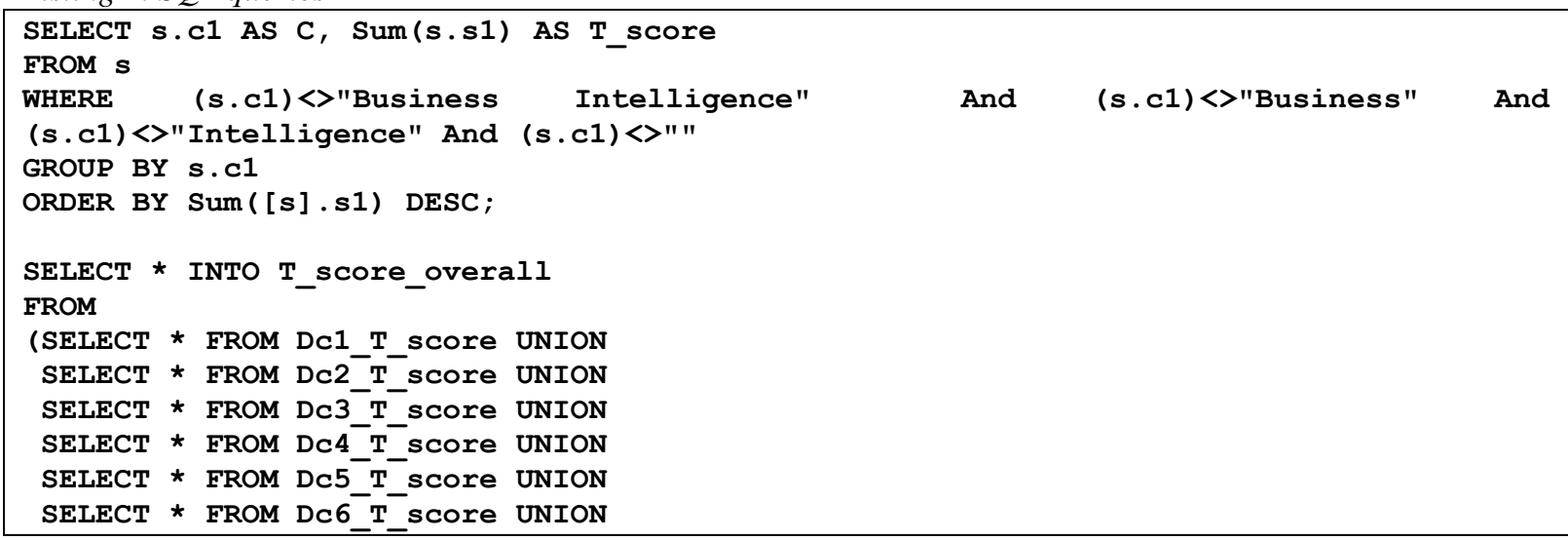




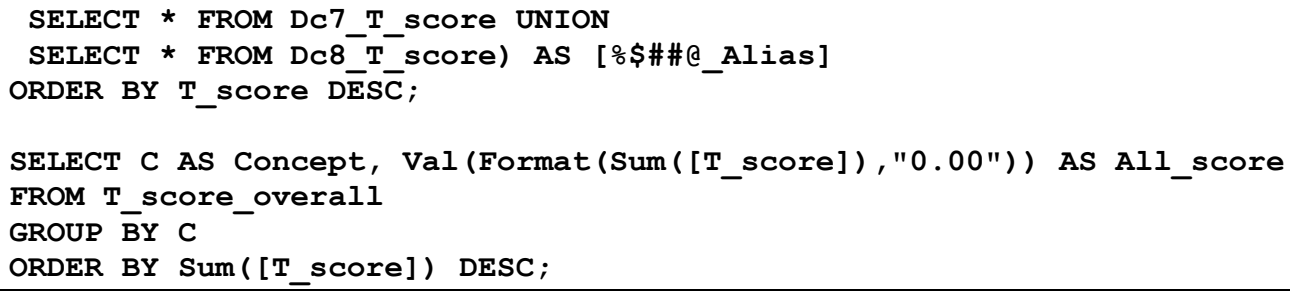

\begin{tabular}{|c|c|c|}
\hline \multicolumn{3}{|c|}{ 圈 Distinct_T_score_overall_plus_score } \\
\hline \multicolumn{2}{|r|}{ Concept } & All_score \\
\hline \multicolumn{2}{|r|}{ Decision theory } & 4428.26 \\
\hline \multicolumn{2}{|r|}{ Decision engineering } & 2394.22 \\
\hline \multicolumn{2}{|r|}{ Knowledge engineering } & 1839.91 \\
\hline \multicolumn{2}{|r|}{ Data warehouse } & 1175.8 \\
\hline \multicolumn{2}{|r|}{ Information systems } & 864.62 \\
\hline \multicolumn{2}{|r|}{ Self service software } & 857.43 \\
\hline \multicolumn{2}{|r|}{ Decision making } & 623.65 \\
\hline \multicolumn{2}{|r|}{ Medicine } & 586.79 \\
\hline \multicolumn{2}{|r|}{ Clinical decision support system } & 558.04 \\
\hline \multicolumn{2}{|r|}{ Management } & 447.42 \\
\hline \multicolumn{2}{|r|}{ Scientific method } & 442.79 \\
\hline \multicolumn{2}{|r|}{ Health care } & 412.6 \\
\hline \multicolumn{2}{|r|}{ Risk } & 353.54 \\
\hline \multicolumn{2}{|r|}{ Artificial intelligence } & 263.68 \\
\hline \multicolumn{2}{|r|}{ Cognition } & 257.93 \\
\hline & Decision making software & 223.79 \\
\hline & Pharmacology & 191.79 \\
\hline & Operations research & 191.75 \\
\hline & Physician & 171.12 \\
\hline & Hospital & 167.37 \\
\hline & Electronic health record & 166.56 \\
\hline & Problem solving & 166.31 \\
\hline & Mathematics & 165.68 \\
\hline & Machine learning & 161.81 \\
\hline & Algorithm & 161.78 \\
\hline & Clinical trial & 153.3 \\
\hline & Agriculture & 152.2 \\
\hline & Water & 148.66 \\
\hline & Logic & 148.31 \\
\hline & Geographic information system & 144.68 \\
\hline $\operatorname{Rec}$ & cord: 14430 of $5147, \mapsto>\mid \nabla_{x}$ & \begin{tabular}{l|l} 
Eilter & Search \\
\end{tabular} \\
\hline
\end{tabular}

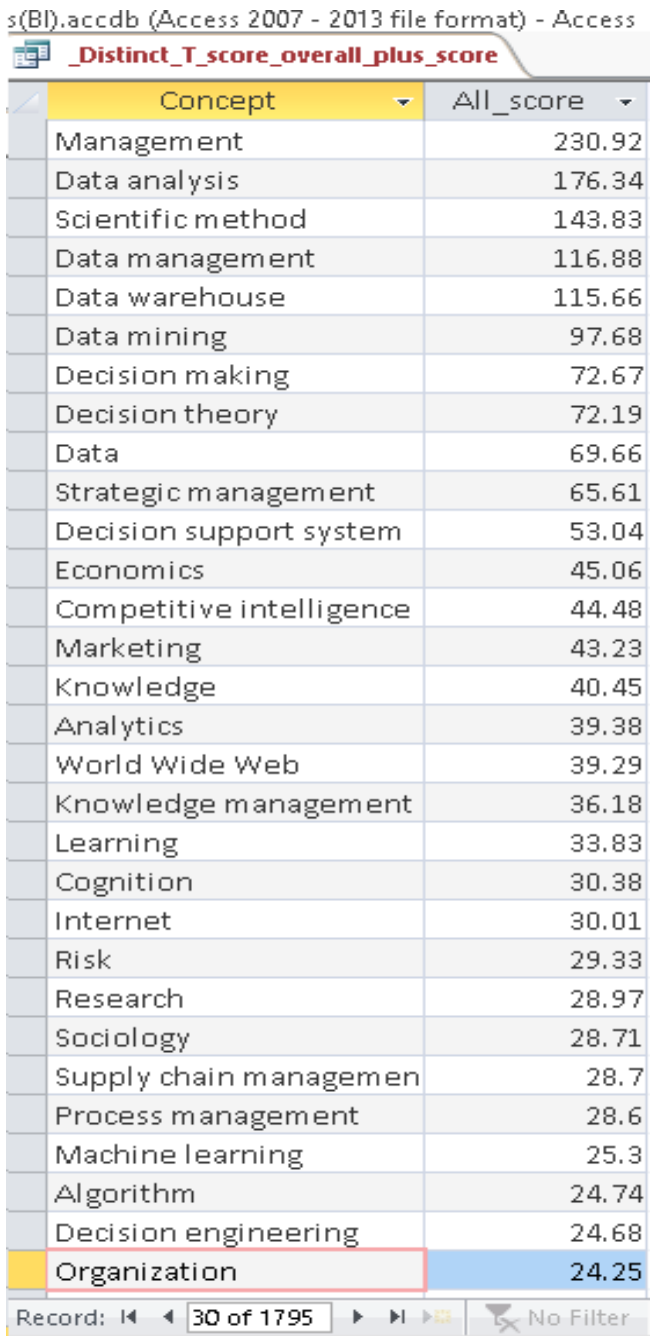

Figure 3. Top 30 most important concepts related to DSS and BI and resulting from the final aggregation

As observed in the figure above (Figure 3), DSS and BI have so many related concepts in common even when considering a restrictive TOP 30 for both. In this case, such common concepts are: Management, Scientific Method, Data Warehouse, Decision Making, Decision Theory, Knowledge, Cognition, Risk, Machine Learning, Algorithm, and Decision Engineering.

\section{Limitations and further ideas and approaches}

The main limitation of our study is due to considering only abstracts together with the titles and keywords and not the full papers which causes possible biases when deriving the most important related concepts using the IBM Watson NLP tool. Some other limitations must be associated with the tool itself. 
www.conferenceie.ase.ro

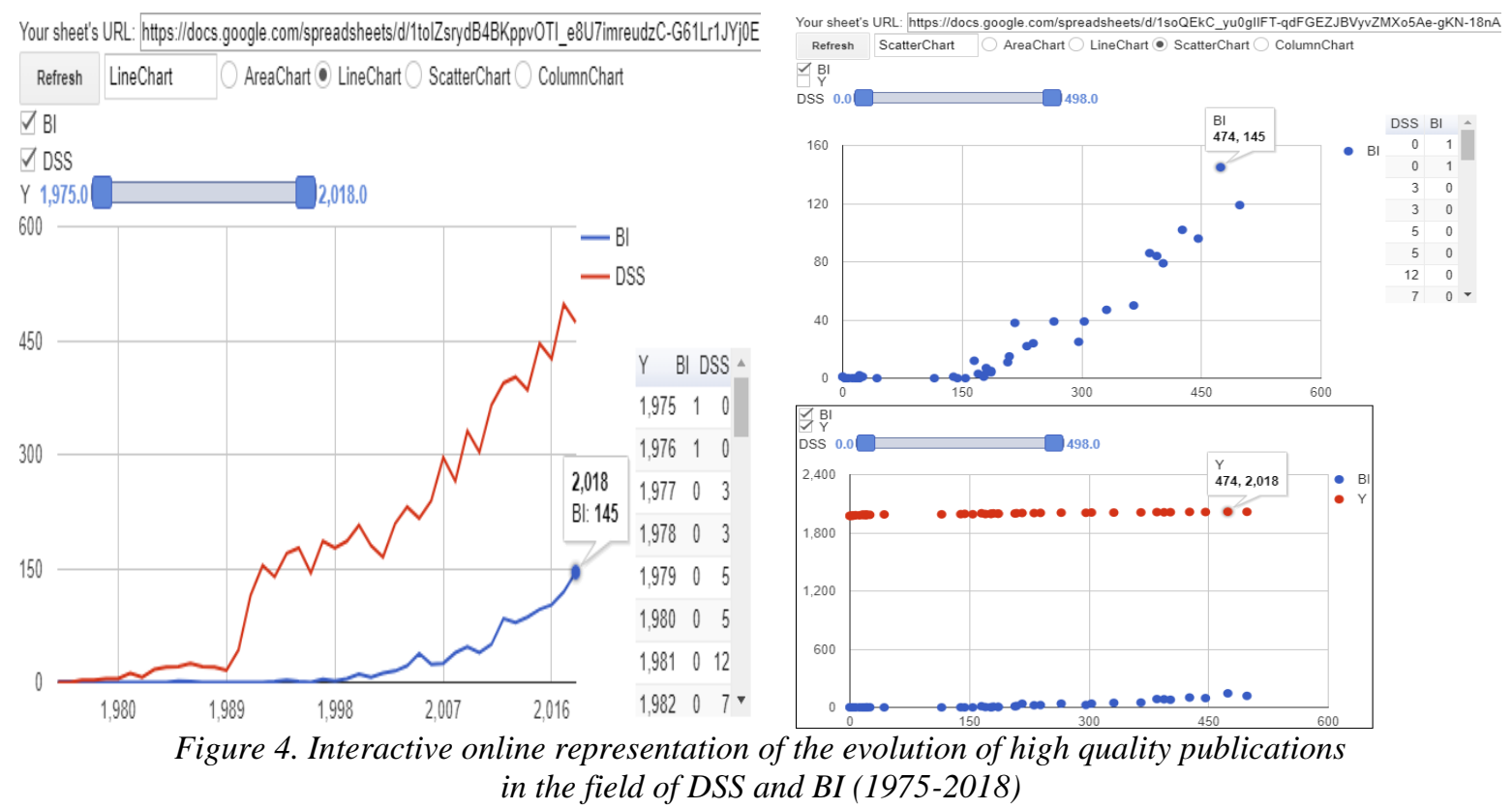

Actually, until now, we built just two time-series corresponding to both starting concepts in this article, namely DSS and BI. In order to create them, we searched again on ISI WOS after those two corresponding exact expressions in extenso, using the same relevance filtering on WOS categories and types of publications and considering only the period between 1975 and 2018. The results were represented in Figure 4 using a cloud-based online interactive plotting tool [7] called GiPlot (sites.google.com/site/supp4giplot/try-it), inspired by an earlier contribution belonging to two of the authors [8]. Both time-series (left of Figure 4 tinyurl.com/y2h7xu4l) show a solid increase in frequency considering the aforementioned period. The correlation diagram (right of Figure 4 - tinyurl.com/y6clxucw) shows at least a cubic if not an exponential interdependence between the evolution of the frequency of publishing high quality papers on the BI topic and the one afferent to the frequency of publishing on the DSS topic.

And that suggests a positive influence exerted by the frequency of high-quality publications related to the more mature field (DSS - also assumed as older) on the one corresponding to the less mature one (BI - also assumed as newer). Anyway, the biggest surprise in figure 4 is not this aforementioned interdependence pattern but it comes when analyzing the starting points of both series (DSS and BI, 1975 and 1976) in the filtered data grids showing that technically speaking BI is the older brother of the two when considering just the 1975-2018 period. Anyway, this additional finding of our paper is enough to contradict the idea [9] [10] [11] [12] that the BI term would have emerged in 1989, being proposed by Howard Dresner.

Next we plan to extract frequency-based time series corresponding to a certain top of those two sets of resulting related concepts to additionally search for as topics (exact expressions) by following the same relevance filters (SCIE, SSCI and the aforementioned publication types) and find more interdependence patterns using forecast options in Data Mining tools.

\section{Conclusions}

Besides discovering hidden patterns behind some topics of interest considered in this article, namely Decision Support Systems and Business Intelligence, the importance of the study is mainly due to the possibility of extending the approach described within to many other interesting topics. We only remind Cloud Computing, Quantum Computing, Internet of Things, Machine Learning, Deep Learning, Big Data and Mobile Applications, easy to search for as 
exact expression on platforms such as Web of Science and objectively conclude about the strongest related concepts as basic support for definitions, classifications, delimitations and further theoretical and practical researches. Some ideas regarding additional derivation of multiple time-series based on the frequency of publications considering related concepts and further analyzes by applying Data Mining tools were also provided.

\section{References}

[1] Z. Panian, "The Evolution of Business Intelligence: From Historical Data Mining to Mobile and Location-based Intelligence", in Proc. WSEAS 4th World Conference of Applied Economics, Business and Development (AEBD), vol. Recent Researches in Business and Economics, 2012, pp. 118-127.

[2] B. Dinter, "The Maturing of a Business Intelligence Maturity Model", in AMCIS 2012 Proceedings, 2012.

[3] T. Chee, L.-K. Chan, M.-H. Chuah, C.-S. Tan, S.-F. Wong and W. Yeoh, "Business Intelligence Systems: State-of-the-Art Review and Contemporary Applications", in Symposium on Progress in Information \& Communication Technology, Kuala Lumpur, Malaysia, 2009.

[4] D. Arnott, "Decision support systems evolution: framework, case study and research agenda", European Journal of Information Systems, vol. 13, no. 4, pp. 247-259, 2004.

[5] T. Nguyen, L. Zhou, V. Spiegler, P. Ieromonachou and Y. Lin, "Big data analytics in supply chain management: A state-of-the-art literature review", Computers \& Operation Research, vol. 98, pp. 254-264, 2018.

[6] D. Arnott and G. Pervan, "A Critical Analysis of Decision Support Systems Research Revisited: The Rise of Design Science", in Enacting Research Methods in Information Systems, Palgrave Macmillan, Cham, 2016, pp. 43-103.

[7] D. Homocianu and M. Homocianu, "GiPlot: An interactive cloud-based tool for visualizing and interpreting large spectral data sets", Spectrochimica Acta Part A: Molecular and Biomolecular Spectroscopy, vol. 209, pp. 234-240, 2019.

[8] D. Homocianu and D. Airinei, "On-Line Dynamic Dashboards in Audit Activities", Audit Financiar, XIII, vol.13, no.125, pp.91-100, 2015.

[9] M. Golfarelli, S. Rizzi and I. Cella, "Beyond data warehousing: what's next in business intelligence", in Proc. The 7th ACM international workshop on Data warehousing and OLAP, Washington, DC, USA, 2004.

[10] S. Rouhani, S. Asgari and S. V. Mirhosseini, "Review Study: Business Intelligence Concepts and Approaches", American Journal of Scientific Research, vol. 50, pp. 62-75, 2012.

[11] I. H. Rajterič, "Overview of Business Intelligence Maturity Models", Journal of Contemporary Management Issues, vol. 15, no.1, pp. 47-67, 2010.

[12] D.J. Power, "Mobile decision support and business intelligence: an overview", Journal of Decision Systems, vol. 22, no.1, pp. 4-9, 2013. 